\title{
Designing Two-Band Mechanical Wave Filters Using Genetic Algorithm
}

\author{
W. Sochacki ${ }^{a}$, J. GARUS $^{a}$, J. SzMIDlA $^{a}$, M. NABIAŁEK $^{b}$,

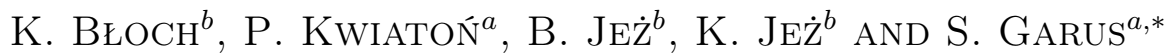 \\ ${ }^{a}$ Department of Mechanics and Fundamentals of Machinery Design, \\ Faculty of Mechanical Engineering and Computer Science, \\ Częstochowa University of Technology, ul. Dabrowskiego 73, 42-201 Częstochowa, Poland \\ ${ }^{b}$ Department of Physics, Faculty of Production Engineering and Materials Technology, \\ Częstochowa University of Technology, ul. Dabbrowskiego 73, 42-201 Częstochowa, Poland \\ Doi: 10.12693/APhysPolA.139.479 \\ *e-mail: s.garus@imipkm.pcz.pl
}

\begin{abstract}
The paper uses a genetic algorithm to design a multilayer structure with two-band gaps in the assumed frequency range of mechanical waves. An appropriate objective function has been designed, allowing for a simultaneous minimization of the transmission from the given frequency ranges and the elimination of high transmission peaks with a small half-width. The paper presents an analysis of the acoustic waves frequency. The transmission of the studied structures was determined using the transfer matrix method algorithm. A single population consisted of 20 structures. In order to minimize the chance of finding solutions to the local minimum, mutations were used in the algorithm and $10 \%$ of random structures were introduced into each population.
\end{abstract}

topics: transfer matrix method, genetic algorithm, phononic multilayers, phononic bandgap

\section{Introduction}

In quasi one-dimensional phononic structures, with the appropriate design (selection of materials, layer thicknesses and distribution of materials in space), there is a phenomenon of a phononic band gap, which means that mechanical waves with certain predetermined frequency regions do not propagate in the structure [1-7] (due to destructive interference). Phononic structures can be used, among others, as sensors, noise suppressors, or selective filters for mechanical waves, $[8,9]$. There are many methods to analyze the properties of phononic structures, such as the finite difference time domain (FDTD) $[10,11]$, the finite element method (FEM) [12], the plane wave expansion (PWE) [13] and the transfer matrix method (TMM) [14] which is used in this work. A genetic algorithm (GA) [15] was used to determine the distribution of layers.

The aim of the work is to develop an algorithm with an appropriately selected objective function so as to minimize the simultaneous transmission in two frequency bands.

\section{Transfer matrix method}

The Thomson-Haskell method, also known as the transfer matrix method, is used to determine the transmission and reflection of quasi one-dimensional structures. In order to determine the transmission, the characteristic matrix of the structure should be defined as

$$
M=T_{1, i}\left(\prod_{i=1}^{n-1} P_{i} T_{i, i+1}\right) P_{n} T_{n, \text { out }} .
$$

It consists of the mechanical wave transmission matrix $T_{i, i+1}$ at the layer boundaries and the propagation matrix $P_{i}$ inside a given layer for the $n$ layer structure. The transmission matrix between the layers $i$ and $i+1$ is influenced by the parameters of the materials from which the layers are made. The acoustic impedance of a given material is defined as $Z_{i}=\rho_{i} c_{i}$. It consists of mass density $\rho_{i}$ and phase velocity $c_{i}$. The latter is determined, in fact, for fluids by the Helmholtz module $c_{i}^{2}=K_{i} / \rho_{i}$, and by the Young modulus $c_{i}^{2}=E_{i} / \rho_{i}$ for solids.

Now, the definition of transmission matrix $T_{i, i+1}$ at the boundary of the layers is

$$
T_{i, i+1}=\frac{1}{2}\left(\begin{array}{ll}
\frac{Z_{i}+Z_{i+1}}{Z_{i}} & \frac{Z_{i}-Z_{i+1}}{Z_{i}} \\
\frac{Z_{i}-Z_{i+1}}{Z_{i}} & \frac{Z_{i}+Z_{i+1}}{Z_{i}}
\end{array}\right) .
$$

The other element included in the characteristic matrix (1) is the propagation matrix $P_{i}$ given by

$$
P_{i}=\left(\begin{array}{cc}
\mathrm{e}^{\mathrm{i} 2 \pi f d_{i} / c_{i}} & 0 \\
0 & \mathrm{e}^{-\mathrm{i} 2 \pi f d_{i} / c_{i}}
\end{array}\right) .
$$

Here, $f$ is the frequency of the incident wave and $d_{i}$ is the thickness of the layer $i$. Substituting the 
matrices (2) and (3) into (1), the characteristic matrix $M$ can be obtained from which the first term of the diagonal can be determined according to

$$
T=\left|M_{11}\right|^{-2} \text {. }
$$

On the basis of the determined transmission for a given frequency range, the components of the objective function used in the genetic algorithm GA are determined.

\section{Genetic algorithm}

In order to determine the optimal structure, a genetic algorithm was used, the individual steps of which are presented in Fig. 1.

At the beginning of the algorithm's operation, a random population is generated consisting of multilayer structures made of the analyzed materials with the parameters presented in Table I [16, 17].

As part of the evaluation stage - the transmission is determined, for each of the structures for the given frequency ranges according to (4). The functions $\left.F_{I}\right|_{f_{\min }} ^{f_{\max }}$ and $\left.F_{D I}\right|_{f_{\min }} ^{f_{\max }}$ are also determined since they constitute the objective functions of the given frequency range $\left.F_{C}\right|_{f_{\min }} ^{f_{\max }}$. The calculations are made with the use of

$$
\begin{aligned}
& \left.F_{I}\right|_{f_{\min }} ^{f_{\max }}=\int_{f_{\min }}^{f_{\max }} \mathrm{d} f T(f) \\
& \approx \frac{f_{\max }-f_{\min }}{2 n} \sum_{i=1}^{n}\left(T\left(f_{i}\right)+T\left(f_{i+1}\right)\right)
\end{aligned}
$$

and

$$
F_{D I}\left|\int_{f_{\min }}^{f_{\max }}=\int_{f_{\min }}^{f_{\max }} \mathrm{d} f\right| \frac{\partial T(f)}{\partial f} \mid .
$$

The component function (5) is responsible for minimizing the transmission in the given frequency band range. In turn, the function (6) composing the objective function of a given frequency band minimizes the occurrence of narrow peaks of high transmission.

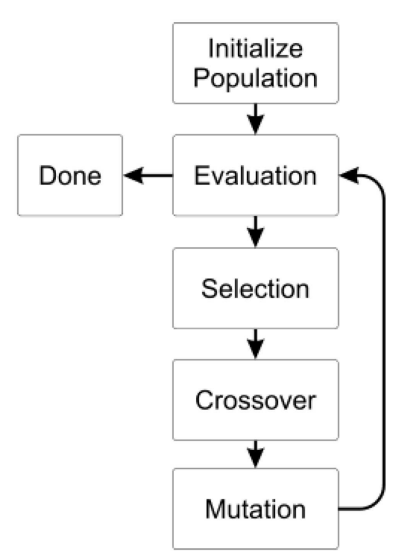

Fig. 1. Individual steps in the search for the best structure using a genetic algorithm.
TABLE I

Properties of materials used in the analyzed multilayer structures [16, 17].

\begin{tabular}{c|c|c|c}
\hline \hline Symbol & Material & $\begin{array}{c}\text { Mass density } \\
\rho\left[\mathrm{kg} / \mathrm{m}^{3}\right]\end{array}$ & $\begin{array}{c}\text { Velocity of } \\
\text { the sound } \\
v[\mathrm{~m} / \mathrm{s}]\end{array}$ \\
\hline B & air & 1.29 & 343 \\
A & $\mathrm{Zr}_{55} \mathrm{Cu}_{30} \mathrm{Ni}_{5} \mathrm{Al}_{10}$ & 66 & 913 \\
& & 6829
\end{tabular}

The objective function for a given frequency range is defined as

$$
\left.F_{C}\right|_{f_{\min }} ^{f_{\max }}=\left\|\left.F_{I}\right|_{f_{\min }} ^{f_{\max }}\right\| \cdot\left\|\left.F_{D I}\right|_{f_{\min }} ^{f_{\max }}\right\| .
$$

As part of our research, the transmission minimizing in two frequency bands, A1 and A2, has been analyzed. We compared two different types of the objective function

$$
F_{S}^{\prime}=F_{\mathrm{A} 1, S}^{\prime}+F_{\mathrm{A} 2, S}^{\prime}
$$

and

$$
F_{P}^{\prime}=F_{\mathrm{A} 1, P}^{\prime} F_{\mathrm{A} 2, P}^{\prime} .
$$

In the first case, the objective function $F_{S}^{\prime}$ consisted of the sum of two functions $F_{\mathrm{A} 1, S}^{\prime}$ and $F_{\mathrm{A} 2, S}^{\prime}$ which were determined from (7) for the analyzed frequency ranges $\mathrm{A} 1$ and $\mathrm{A} 2$, respectively. The function $F_{P}^{\prime}$ was determined analogously, but the component functions were multiplied by each other.

The objective function was first sorted of the given population. Then, the obtained structures were crossed in such a way that the best two structures were left unchanged and the two worst ones were replaced randomly.

In order to minimize the chance of finding solutions to the local minimum, the structures were mutated, except for the two best ones. The cycle was repeated until a given number of iterations was obtained.

\section{Results and discussion}

The aim of the work was to minimize the transmission of a mechanical wave simultaneously in two frequency ranges through a multilayer structure specially designed with the use of a genetic algorithm. The A1 band was in the frequency range from $3 \mathrm{kHz}$ to $5 \mathrm{kHz}$, while the $\mathrm{A} 2$ band was in the range from $8 \mathrm{kHz}$ to $10 \mathrm{kHz}$. One hundred layered structures were analyzed in the study, and each population consisted of 20 individuals. Single layer thickness was $1 \mathrm{~mm}$. The chance of mutating an individual layer in the structure was $1 \%$. The solution space of $2^{100}$ structures was analyzed. The materials used were selected so that there were significant differences in acoustic impedance. For each objective function, 100 iterations of the genetic algorithm were performed. 

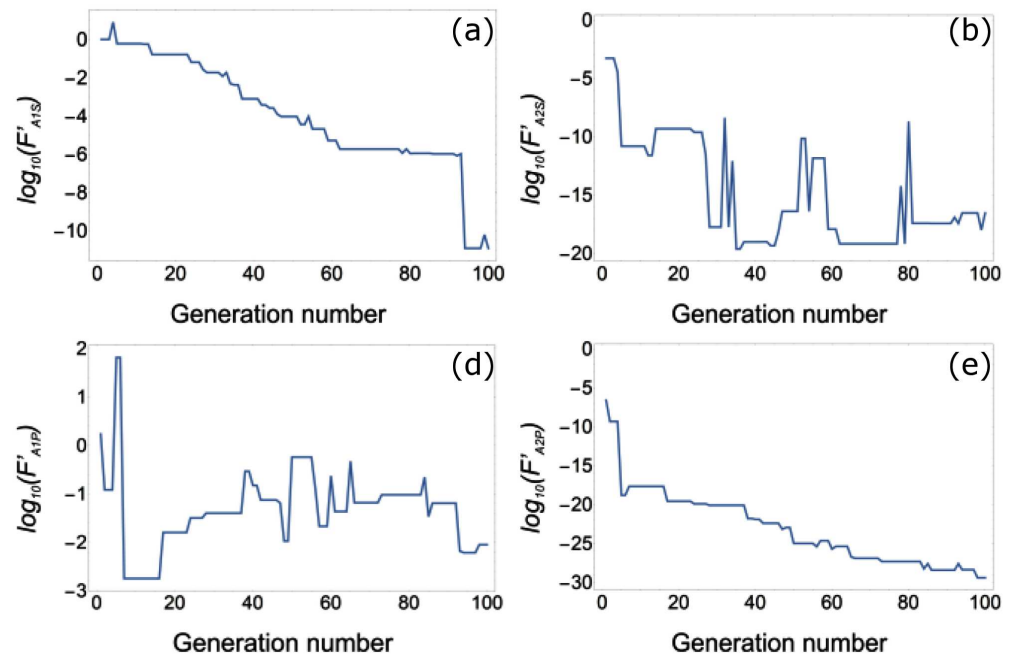

(b)

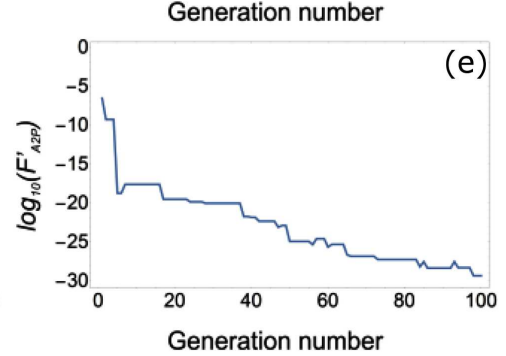

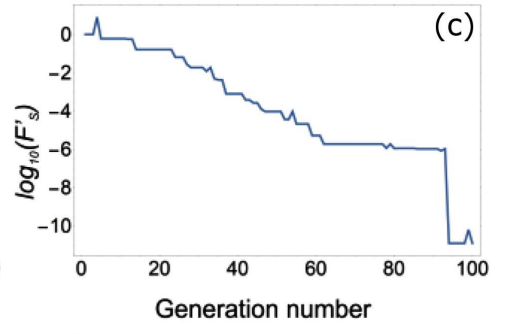

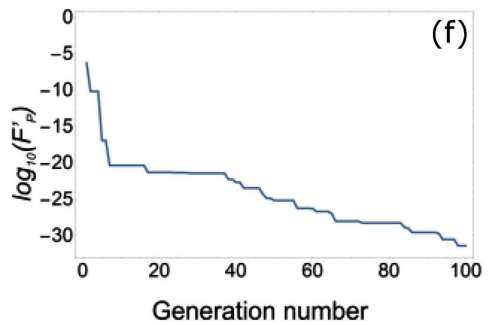

Fig. 2. The objective function values of the best individuals (without normalisation) for each generation. The figures show, respectively, the components for the areas A1 (a) and A2 (b) of the objective function composed of the sum of sub-functions $F_{S}^{\prime}$ (c) and sub-functions for the areas A1 (d) and A2 (e) for the objective function composed of the product of sub-functions $F_{P}^{\prime}$ (f).
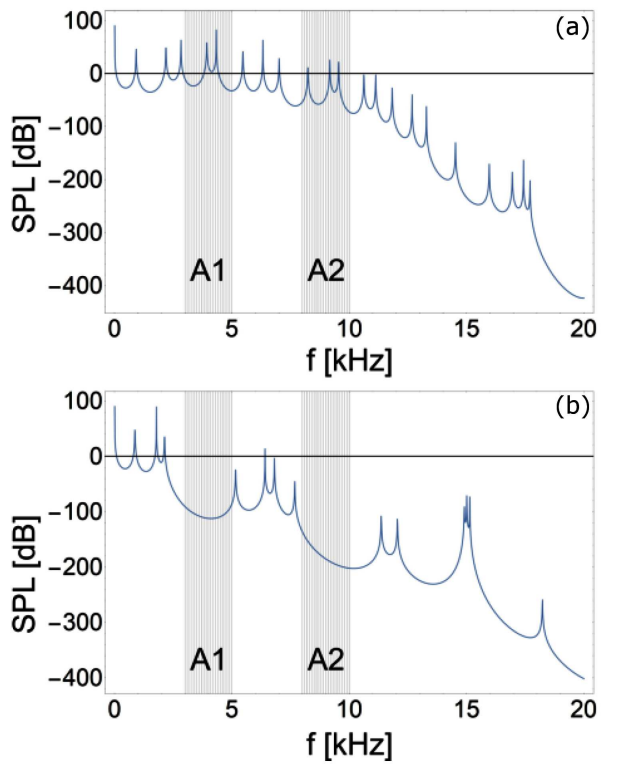

Fig. 3. Transmission for the worst (a) and best (b) analyzed structures for $F_{S}^{\prime}$ objective function.

Graphs are shown on a logarithmic scale for better readability. The best structure found for $F_{P}^{\prime}$ objective function was $\mathrm{A}_{11} \mathrm{~B}_{3} \mathrm{~A}_{3} \mathrm{BA}_{4} \mathrm{~B}_{6} \mathrm{ABA}_{4} \mathrm{~B}_{12} \mathrm{~A}_{7}$ $\mathrm{B}_{4} \mathrm{~A}_{6} \mathrm{BABA}_{10} \mathrm{~B}_{4} \mathrm{~A}_{3} \mathrm{~B}_{7} \mathrm{~A}_{7} \mathrm{~B}_{3}$. The best structure for $F_{S}^{\prime}$ was $\mathrm{B}_{4} \mathrm{AB}_{2} \mathrm{~A}_{2} \mathrm{BABABABA}_{2} \mathrm{~B}_{6} \mathrm{ABA}_{2} \mathrm{~B}_{4} \mathrm{~A}_{10} \mathrm{~B}_{9}$ $\mathrm{AB}_{9} \mathrm{~A}_{2} \mathrm{BA}_{6} \mathrm{BA}_{2} \mathrm{BABAB}_{11} \mathrm{~A}_{8} \mathrm{BAB}_{2}$. The subscript specifies the number of repetitions of a given layer. Figure 2 shows how the analyzed objective functions and their components changed for the best analyzed structures in each iteration. The graphs do not take into account the normalization of the components of the objective functions, and the results for better readability are presented on a logarithmic scale. For the target function $F_{S}^{\prime}$, it can be seen that the component of the objective function for the A2 area very quickly reached a low value, while the greater part of the minimization process took place for the A1 area.

Figures 3 and 4 show the transmissions for the worst and best analyzed structures, respectively, for the objective function with the sum of sub-functions and their multiplication. Transmission was minimized in the shaded areas. The reference pressure value was $2 \times 10^{-5} \mathrm{~Pa}$ and it is marked on the graphs by a horizontal line as $0 \mathrm{~dB}$. The maximum value of the sound pressure level(SPL) was $90 \mathrm{~dB}$. The SPL below $0 \mathrm{~dB}$ can be considered as a gap. It should be

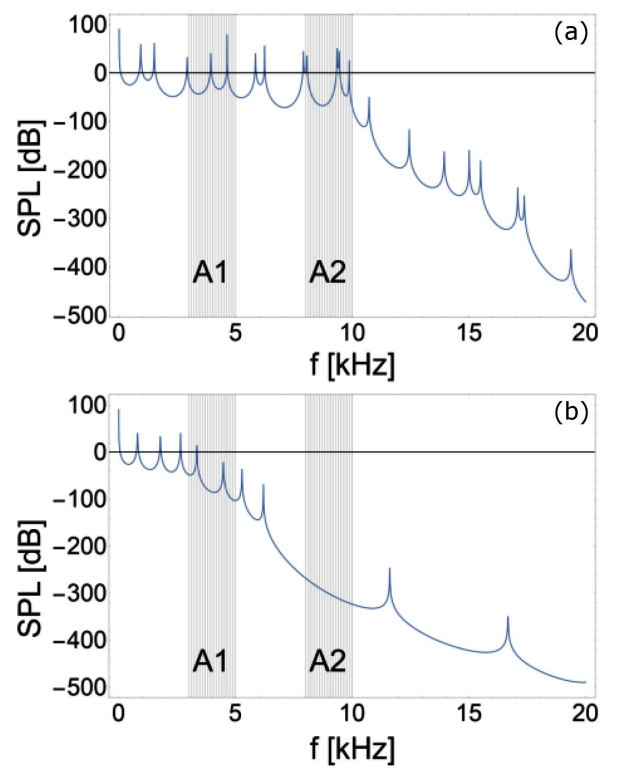

Fig. 4. Transmission for the worst (a) and best (b) analyzed structures for $F_{P}^{\prime}$ objective function. 

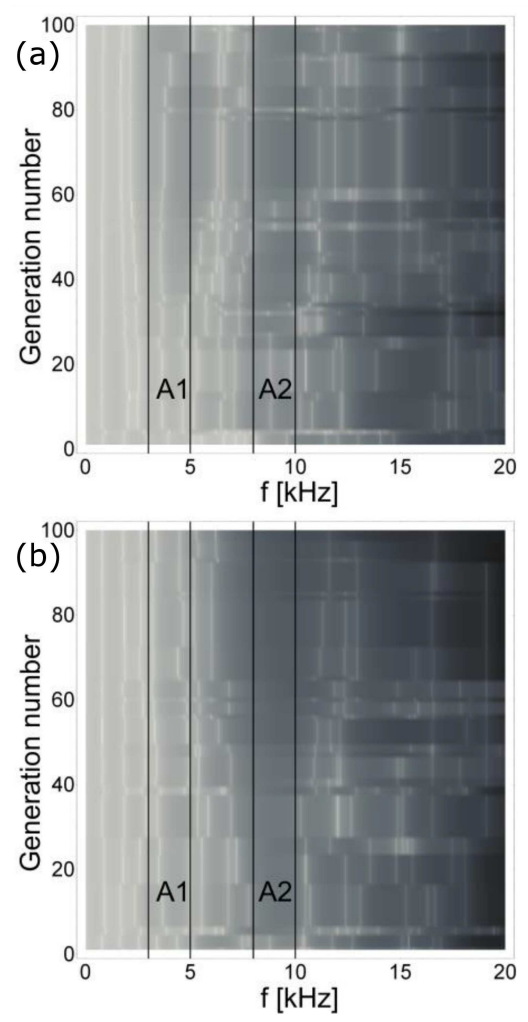

Fig. 5. The density plots with transmission for the best individuals for each generation in searches for $F_{S}^{\prime}(\mathrm{a})$ and $F_{P}^{\prime}(\mathrm{b})$.

noted that the genetic algorithm with the objective function consisting of the sum of sub-functions in the entire frequency range of the A1 and A2 bands showed transmission below $0 \mathrm{~dB}$, while in $\mathrm{A} 1$ for the second objective function there was a single narrow bandwidth.

Figure 5 shows the transmission SPL $[\mathrm{dB}]$ of the best analyzed structures in each population. The white color means full transmission, while the black color means no transmission through the given structure. Despite the presence of the band gap almost from the beginning of the algorithm's operation in the A2 area for the function $F_{P}^{\prime}$, at the end of the algorithm's operation there was a single transmission band in the A1 area.

\section{Conclusions}

The paper shows that it is possible to use a genetic algorithm to search for multilayer structures with given transmission properties. The algorithm allowed to find multilayer structures built of amorphous alloy and PVC for two frequency bands where the objective function was to reduce transmission and eliminate high transmission peaks with a small half width. Such structures can be used as mechanical wave filters and noise control devices.

In the work, finding the optimal assumed structure took less than 100 iterations of the algorithm. Two objective functions consisting of sub-functions determined for each area were analyzed. For both cases, the algorithm determined structures with significantly lower transmission. Better effects were seen for the objective function consisting of the sum of sub-functions where all transmission peaks were eliminated.

\section{Acknowledgments}

The study has been carried out within statutory research of the Department of Mechanics and Machine Design Fundamentals of the Częstochowa University of Technology.

\section{References}

[1] I. Kriegel, F. Scotognella, Physica E 85, 34 (2017).

[2] Y.F. Li, F. Meng, S. Li, B. Jia, S. Zhou, X. Huang, Phys. Lett. A 382, 679 (2018).

[3] Y. Pennec, B. Djafari-Rouhani, H. Larabi, J. Vasseur, A.-C. Hladky-Hennion, Phys. Status Solidi C 6,2080 (2009).

[4] M.S. Kushwaha, Int. J. Mod. Phys. B 10, 977 (1996).

[5] Y. Tanaka, T. Yano, S-i. Tamura, Wave Motion 44, 501 (2007).

[6] M. Sigalas, E.N. Economou, J. Sound Vib. 158, 377 (1992).

[7] S. Kushwaha, P. Halevi, L. Dobrzynski, B. Djafari-Rouhani, Phys. Rev. Lett. 71, 2022 (1993).

[8] I. Kriegel, F. Scotognella, Physica E 85, 34 (2017).

[9] S. Villa-Arango, R. Torres, P.A. Kyriacou, R. Lucklum, Measurement 102, 20 (2017).

[10] A. Taflove, Computational Electrodynamics: The Finite-Difference Time-Domain Method, Artech House, Norwood (MA) 1995.

[11] D.M. Sullivan, Electromagnetic Simulation Using the FDTD Method, IEEE Press, New York 2000.

[12] D.L. Dwoyer, M.Y. Hussaini, R.G. Voigt, Finite Elements - Theory and Application, Springer-Verlag, New York 1986.

[13] S.G. Johnson, J.D. Joannopoulos, Opt. Expr. 8, 173 (2001).

[14] M.N. Armenise, C.E. Campanella, C. Ciminelli, F. Dell'Olio, V.M.N. Passaro, Phys. Proced. 3, 357 (2010).

[15] S. Garus, W. Sochacki, Wave Motion 98, 102645 (2020).

[16] Shu Yang, Wei-Dong Yu, Ning Pan, Physica B: Condens. Matter 406, 963 (2011).

[17] M. Fukuhara X. Wang, A. Inoue, J. NonCryst. Solids 356, 1707 (2010). 